\title{
A HER2-Target Antibody Drug Conjugate Combined with anti-PD-(L)1 Treatment Eliminates hHER2 + Tumors in hPD-1 Transgenic Mouse Model and Contributes Immune Memory Formation
}

Lei Huang ( $\sim 1510776 @$ @tongji.edu.cn )

Tongji University https://orcid.org/0000-0001-6187-8683

Ruiqin Wang

Tongji University

Kun Xie

Tongji University

jingming Zhang

Tongji University

Fei Tao

Tongji University

Chenyu Pi

Tongji University

Yan Feng

Tongji University school of life sciences and technology

Jianmin Fang

Tongji University

Hua Gu

Tongji University https://orcid.org/0000-0002-4442-9808

\section{Research Article}

Keywords: Antibody-drug conjugates, HER2-positive breast cancer, checkpoint inhibitor combination therapy

Posted Date: July 8th, 2021

DOl: https://doi.org/10.21203/rs.3.rs-534358/v1

License: (c) (1) This work is licensed under a Creative Commons Attribution 4.0 International License. Read Full License 
Version of Record: A version of this preprint was published at Breast Cancer Research and Treatment on October 16th, 2021. See the published version at https://doi.org/10.1007/s10549-021-06384-4. 


\title{
A HER2-target antibody drug conjugate combined with anti-PD-(L)1 treatment eliminates hHER2 + tumors in hPD-1 transgenic mouse model and contributes immune memory formation
}

\author{
Author names \\ Lei Huang ${ }^{1}$, Ruiqin Wang ${ }^{1}$, Kun Xie ${ }^{1}$, Jingming Zhang ${ }^{1}$, Fei Tao ${ }^{1}$, Chenyu $\mathrm{Pi}^{1}$, Yan Feng ${ }^{1}$, Jianming Fang ${ }^{1,2,3^{*}}$, Hua \\ $\mathrm{Gu}^{1, *}$
}

Affiliations

1 Laboratory of Molecular Medicine, Shanghai Key Laboratory of Signaling and Disease Research, School of Life Sciences and Technology, Tongji University, Shanghai 200092, People's Republic of China;

2 Department of Neurology, Tongji Hospital, Tongji University, Shanghai, People's Republic of China;

${ }_{3}$ Biomedical Research Center, Tongji University Suzhou Institute, Suzhou, Jiangsu, People's Republic of China

*Corresponding author: Hua Gu ${ }^{*}$, Jianming Fang *

Email:

\begin{abstract}
Purpose: Disitamab vedotin (RC48) is an HER2-directed antibody-drug conjugate, emerging as an effective strategy for cancer therapy, not only enhance antitumor immunity in previous animal models but also improve clinical outcomes for patients such as with gastric cancer, urothelium carcinoma and HER2 low-expressing breast cancer. Here, we explore the combination therapeutic efficacy of this novel HER2-targeting ADC with immune checkpoint inhibitors in a human HER2-expressing syngeneic breast cancer model.

Methods: The human HER2 + cancer cell line is constructed by stably transfection and individual clones were isolated by single-cell sorting. Flow cytometry was performed to determine its binding activity. Cytotoxic effect was determined using an MTT assay with the supplement of RC48. Human PD-1 transgenic mice were used to analyze the in vivo anti-tumor effects of the ADC and its combination therapy with PD-1/PD-L1 antibody.

Results: The combination of RC48 and PD-1/PD-L1 immune checkpoint inhibition significantly enhanced tumor suppression and antitumor immunity. Tumor rejection in the synergistic groups was accompanied by massive T-cell infiltration and immune marker activation. Furthermore, the combination therapy promoted immunological memory formation in the tumor-eradication animals, protecting them from tumor rechallenge. Conclusion: A novel HER2-targeting ADC combined with immune checkpoint inhibitors can achieve remarkable effects in mice and elicit long-lasting immune protection in a hHER2+ murine breast cancer model. This study provides insights into the efficacy of RC48 therapeutic activity and a rationale for potential therapeutic combination strategies with immunotherapy.
\end{abstract}

Keywords: Antibody-drug conjugates, HER2-positive breast cancer, checkpoint inhibitor combination therapy 


\section{Introduction}

Amplification and overexpression of human epidermal growth factor receptor 2 (HER2) are commonly observed in human breast cancers and often associated with poor patient survival ${ }^{1-5}$. This genetic alteration promotes cancer cell proliferation and survival, suggesting that HER2 is a promising therapeutic target for cancers. Trastuzumab is one of successful monoclonal antibodies against HER2, which has been clinically proven to be significantly efficient in multiple indications ${ }^{6,7}$. Nonetheless, de novo and acquired resistance to HER2 blockade eventually occurs in most patients with advanced disease. Therefore, new strategies are clearly needed for HER2-positive breast cancer, which to date remains poor clinical outcomes.

Antibody-drug conjugate (ADC) drugs are loaded with a small toxin molecule and exploit the function of monoclonal antibodies, which can enrich a large number of toxin molecules around the targeted tumor cells, thereby exerting a more powerful killing effect ${ }^{8-11}$; for example, ado-trastuzumab emtansine (T-DM1) $)^{12}$ and trastuzumab deruxtecan (DS-8201a) ${ }^{13}$, which were approved by the FDA in 2013 and 2019, both use trastuzumab as the antibody backbone. Another promising ADC is disitamab vedotin $(\mathrm{RC} 48)^{14,15}$, which combines disitamab-novel HER2 target specificity — with a potent microtubulin polymerization inhibitor monomethyl auristatin E (MMAE) as a payload, allowing for targeted drug delivery. Given that resistance often occurs in patients receiving ADC therapy post treatment. Likewise, despite the favorable efficacy results, most patients treated with T-DM1 eventually progress in HER2-positive breast cancers ${ }^{16}$. Moreover, ADCs reduce the number of targeted tumor cells, thereby minimizing the exposure of tumor-derived antigens, which may render them nonfunctional ${ }^{17}$.

In previous studies, immune-mediated mechanisms for microtubule-depolymerizing agents have been reported, such as dolastatin-10 and its synthetic analog $M M A E^{18}$. Brentuximab vedotin is a representative of using MMAE as a cytotoxic payload, which results in sustained clinical responses in patients with CD30+ lymphomas $^{19}$. Indeed, brentuximab vedotin induces activation of patient DCs, T cells, and B cells, reflecting augmentation of tumor-specific immunity. Given the observation of immune engagement for the microtubule-depolymerizing cytotoxic moieties used in ADCs, and chemotherapeutic agents induced tumor cell death ${ }^{20}$, we hypothesized that the clinical activity observed with RC48 partly relies on an efficient antitumor immune response. The latter would allow the combination of RC48 treatment with immunotherapeutic strategies such as immune checkpoint inhibitors, including programmed cell death protein 1 (PD-1) and its ligand (PD-L1), which may help overcome resistance and potentially result in long-term benefit.

To explore the therapeutic response of the combination of RC48 with immune checkpoint inhibition, we employed a human (h) HER2+ syngeneic breast cancer model and humanized immune checkpoint inhibitors in our transgenic hPD-1 mouse model.

\section{Material and Methods}

\section{Antibodies and compounds}

Disitamab vedotin (RC48) and its parental anti-human HER2 (anti-hHER2) antibody were prepared as previously described ${ }^{14,15}$. The drug-to-antibody ratio was 4 , as determined by hydrophobic interaction chromatography.

For flow cytometry, fixable viability stain 450 (562247; BD Biosciences, Franklin Lakes, NJ), PercP/Cy5.5-labeled anti-mouse CD45 (30-F11, 550994; BD Biosciences), APC-labeled anti-mouse CD3e (100236; BioLegend, San 
Diego, CA), FITC-labeled anti-mouse CD4 (100405; BioLegend), FITC-labeled anti-mouse CD8a (100705; BioLegend), and APC-labeled anti-mouse CD274 (B7-H1, PD-L1) (124312; BioLegend) antibodies were used.

\section{Cell lines and cell culture}

The human breast cancer cell line BT474, mouse triple-negative breast cancer cell line E0771, and 293T cells were purchased from the American Type Culture Collection (Manassas, VA) and cultured in Dulbecco's modified Eagle medium (Hyclone, Waltham, MA) supplemented with 10\% fetal bovine serum (Cegrogen Biotech, Stadtallendorf, Germany), $100 \mathrm{U} / \mathrm{mL}$ penicillin (Gibco, Waltham MA), and $100 \mu \mathrm{g} / \mathrm{mL}$ streptomycin (Gibco) at $37{ }^{\circ} \mathrm{C}$ and $5 \% \mathrm{CO}_{2}$. All cell lines were tested for no contamination.

\section{Construction of the hHER2+ mouse cancer cell line}

E0771-hHER2 is a murine breast cancer cell line expressing hHER2. Human full-length HER2 cDNA from BT474 was cloned into the transposon-based vector PLVX-IRES-ZsGreen1 and co-transfected into murine wild-type (WT) E0771 cells together with PMD2.G and psPAX2 for four rounds. All the vectors were maintained in our lab and proved for sequences. Then, cell pools stably expressing hHER2 were selected in the presence of puromycin, and individual clones were isolated by single-cell sorting using flow cytometry (FACS Aria II; BD Biosciences).

\section{Cell counting kit (CCK)-8}

E0771-hHER2 cells $\left(5 \times 10^{3}\right)$ were plated in 96 -well plates, after which RC48 $\left(0,10^{-4}, 10^{-3}, 10^{-2}, 10^{-1}, 10^{\circ}\right.$, and 10 $\mu \mathrm{g} / \mathrm{mL}$ ) was added to the wells (4 replicate wells per concentration). After $96 \mathrm{~h}$, metabolic activity was determined using CCK-8 assay (HY-K0301; MCE, New Jersey). IC50 values were calculated using GraphPad Prism v6 (GraphPad Software, Inc., La Jolla, CA) using a four-parameter logistic (4PL) regression model.

\section{Flow cytometry}

E0771 WT and E0771-hHER2 $\left(1 \times 10^{6}\right.$ cells $\left./ \mathrm{mL}, 100 \mu \mathrm{L}\right)$ were separately treated on ice for $1 \mathrm{~h}$ with the indicated concentration $(0.0001-100 \mu \mathrm{g} / \mathrm{mL})$ of disitamab, followed by incubation for $30 \mathrm{~min}$ with $\operatorname{lgG}$ Fc-APC (409306; BioLegend) and fluorescence-activated cell sorting (FACSCalibur; BD Biosciences). Data were analyzed using FlowJo 7.6.2 software (TreeStar, Inc., Ashland, OR).

\section{Immunofluorescence staining}

For tumor immunofluorescence staining, samples were fixed immediately after excision in $4 \%$ paraformaldehyde overnight at $4{ }^{\circ} \mathrm{C}$. Excised tumor tissues were frozen using OCT compound (4583; Solarbio, Beijing, China) and sectioned for immunohistochemical staining of CD3, and Ki67 (6B111141, Servicebio, Wuhan, China).

\section{Mouse models and treatments}

All procedures relating to animal care, handling, and treatment were performed according to the European Union Directive 2010/63/EU and approved by the Animal Research Ethics Committee at Tongji University (no. TJLAC-018-032). Human PD-1 transgenic mice were obtained from Shanghai Model Organisms Co., Ltd. (Shanghai, China) and kept in a pathogen-free environment. Mice (aged 5 to 6 weeks) were inoculated with 2 $\times 10^{6}$ E0771-hHER2 cells suspended in PBS into the right flank by subcutaneous (s.c.) injection. Tumor volume was defined as $1 / 2$ length $\times$ width $^{2}$. When the average volume of tumors reached approximately 100-200 $\mathrm{mm}^{3}$, the mice were randomized divided into control and treatment groups based on tumor volumes, and treatment was initiated (day 0). RC48 (10 mg/kg), anti-hHER2 antibody (10 mg/kg), and vehicle (PBS) were 
administered intravenously (i.v.) at a volume of $100 \mu \mathrm{L} /$ mouse. Tumor volume was analyzed by two-way ANOVA using GraphPad Prism v6. Mouse body weight was determined using an electronic scale.

In drug combination experiments, mice were randomly divided into 6 groups with similar mean tumor sizes, and treated with PBS, ADC (5 mg/kg), PD-1 antibody (10 mg/kg; Pembrolizumab; Merck \& Co., Kenilworth, NJ), PD-L1 antibody (10 mg/kg; Atezolizumab, Roche, Mannheim, Germany), or their combination (ADC + PD-1 antibody or ADC + PD-L1 antibody). RC48 was administered on days 0 and 7. Anti-PD-1 and anti-PD-L1 antibodies were administered on days $0,3,7$, and 10.

For the rechallenge study, mice E0771-hHER2 tumors cured by RC48 and PD-1/PD-L1 treatment were divided into two groups. The mice were then s.c. inoculated with $2 \times 10^{6}$ E0771-WT or E0771-hHER2 cells into the left flank. Naive mice (previously not inoculated with tumor cells) were also inoculated with each cell line for comparison.

\section{Tumor digestion and analysis of intratumoral cells}

On day 7 after treatment initiation, mice were euthanized, and tumors were mechanically dissociated and digested with 0.1\% collagenase type I (17100-017; Invitrogen, Carlsbad, CA) supplemented with Hanks' balanced salt solution medium (HBSS; 2005368; Gibco) for $20 \mathrm{~min}$ at $37{ }^{\circ} \mathrm{C}$. Primary cells were harvested, washed, and resuspended in HBSS containing $1 \%$ bovine serum albumin. Single-cell suspensions were prepared and stained with the indicated markers for flow cytometry. The resultant single cells were stained with live/dead agent, and followed with antibodies against mouse CD3, CD4, CD8, CD45, NK1.1, and PD-L1.

\section{Statistical analysis}

Statistical analysis was performed using GraphPad Prism v6. Results are shown as the mean \pm SEM of at least triplicate technical or biological replicates. Statistical significance was analyzed by an unpaired two-tailed $t$-test unless otherwise indicated. Differences were considered significant at $p<0.05$.

\section{Results}

\section{RC48 decreases hHER2+ cancer cell viability}

To study on RC48 and the parental anti-hHER2 antibody which does not cross-react with mouse HER2, we constructed a mouse cell line that stably expresses the human target gene. The human her2 gene was introduced into the murine triple-negative breast cancer cell line E0771 using the pLVX-IRES-ZsGreen1 plasmid. After four rounds of fluorescent-activated cell sorting (FACS), cells with high hHER2 expression levels were obtained, which bound to anti-hHER2 antibody. Monoclonal culture was conducted, after which clone \#33 with the highest expression level was selected and named E0771-hHER2 in subsequent animal experiments (Fig. 1a). A series of concentration gradients of anti-hHER2 antibody staining were used to determine the binding ability of E0771-hHER2 (Fig. 1b).

In in vitro cell viability assays of E0771-hHER2 after RC48 treatment, the IC50 value was determined to be 90 $\mathrm{ng} / \mathrm{mL}$ (Fig. 1c). Subsequently, E0771-hHER2 cells treated with RC48 (0, 100, and $500 \mathrm{ng} / \mathrm{mL})$ for $48 \mathrm{~h}$ and $72 \mathrm{~h}$ were imaged. Off-target effects of RC48 were assessed on breast cancer lines lacking expression of hHER2 (E0771 WT). Compared with E0771 WT, E0771-hHER2 cell viability was significantly reduced by RC48. (Fig. 1d). These results indicate that the E0771-hHER2 cell line can be used for further studies. 


\section{RC48 treatment increases intratumoral T cell infiltration}

Previous reports revealed that the MMAE molecule of ADC drugs is a toxin molecule that results in immunogenicity $^{18}$. To figure it out, E0771-hHER2 cells were s.c. inoculated into PD-1 mice, and tumor growth was confirmed. When mice with E0771-hHER2 tumors were i.v. treated with vehicle, $10 \mathrm{mg} / \mathrm{kg}$ of RC48, or 10 $\mathrm{mg} / \mathrm{kg}$ of its parental anti-hHER2 antibody on day 5, the mean tumor volumes on day 9 of vehicle-, anti-hHER2 antibody-, and RC48-treated groups were $345.07 \pm 78.00,358.83 \pm 107.69$, and $244.82 \pm 78.90 \mathrm{~mm}^{3}$, respectively (Fig. 2a). Compared with the other two groups, RC48 showed a better antitumor effect, indicating that the antitumor effect of RC48 in this model depends mainly on the payload delivered into the tumor by anti-hHER2 antibody.

Next, the tumor was excised on day 9, and immunofluorescence staining was performed. It was found that the RC48 group exhibited increased infiltrated T cells in the tumor tissue compared with that of the parental monoclonal antibody (Fig. 2b), which may have induced an immune response. This indicates the potential for treatment combinations with immune checkpoint inhibitors.

\section{RC48 and PD-1/PD-L1 combination treatment shows improved tumor inhibition}

As immune cell infiltration was observed in mouse tumors after RC48 therapy, we speculated that adding an immune checkpoint inhibitor can lead to synergistic therapeutic effects. Therefore, we assessed the combination of RC48 with anti-PD-1 or anti-PD-L1 antibodies in an immunocompetent mouse model with E0771-hHER2 (s.c. inoculation). When mice were treated with vehicle (PBS), RC48 (5 mg/kg, once a week, 2 times, i.v.), anti-PD-L1 antibody (15 mg/kg, twice a week, 2 cycles, i.v.), or a combination of the two, tumor growth after monotherapy was slower than that of the PBS group; meanwhile, tumors in the combination group disappeared within two or three weeks, showing a very significant combined outcome (Fig. 3a). In the RC48+anti-PD-1 group (10 mg/kg, twice a week, 2 cycles, i.v.), the same result of significant combined outcome was also obtained (Fig. 3b). Thus, the combination of PD-1 or PD-L1 antibody with ADC achieved a combinatorial effect. Individual tumor growth curves for each group are shown in Fig. 3c, and the standard error in the RC48 + anti-PD-L1 treatment group was negligible.

\section{Characterization of intratumoral immune cells after RC48 + PD-1/PD-L1 combination therapy}

To elucidate the rapid shrinkage of tumors in the combination therapy group, we analyzed the infiltrated immune cells in tumors of mice injected with ADC and/or PD-1/PD-L1 antibodies. After 7 days of treatment, the proportion of CD45 in bulk tumors from the two combination groups was significantly higher than that in the monotherapy group (Fig. 4a, Fig.S2). The proportion of $\mathrm{CD}^{+}, \mathrm{CD}^{+}, \mathrm{CD}^{+}$and NK cells in the combination groups was also higher than in the vehicle control and monotherapy group (Fig. 4b-e).

To examine whether RC48, PD-1/PD-L1, or a combination of the two modulates the expression of immune-associated markers on tumor cells, PD-L1 expression levels were determined by FACS (Fig. 4f). Interesting, the median fluorescence intensity of PD-L1 on hHER2+ cells as well as total cells from the excised tumors was higher in the anti-PD-1 therapy (both mono- and combo-) group than other groups (Fig. 5g). Otherwise, both anti-PD-1 and anti-PD-L1 therapy decreased the PD-L1 expression on the surface of the infiltrating $\mathrm{CD}_{4} 5^{+}$immune cells. Therefore, our data reveal an immune-based mechanism of action for combining RC48 with immune checkpoint inhibition.

\section{Tumor immunostaining after RC48 + PD-1/PD-L1 combination therapy}

Histomorphological evaluation of tumors after 7 days of RC48 monotherapy and RC48 + immune checkpoint 
inhibitors revealed no histologically evident treatment effect in the tumor cell compartment. Quantitative changes indicated that expression of Ki67-a tumor proliferation marker—was decreased (Fig. 5a-b). These findings demonstrate the direct anti-tumor microenvironment of combining RC48 with immune checkpoint inhibitors.

\section{Contribution of RC48+PD-1/PD-L1 combination treatment to immune memory formation}

A complete response was observed in 100\% of E0771-hHER2 (s.c. inoculation)-bearing mice treated with RC48+PD-1 and RC48+PD-L1. These cured mice were randomly divided into different groups and rechallenged with E0771-hHER2 or E0771-WT cells (s.c. inoculation). Naive mice were used as a control cohort (Fig. 6a).

E0771-hHER2 cells were completely rejected in rechallenged mice. Furthermore, E0771-WT cells were also rejected, albeit to a lesser extent, in mice cured of the E0771-hHER2 tumor (Fig. 6b, Fig. S3). Meanwhile, E0771-hHER2 and E0771-WT cells grew normally in naive mice. These results suggest that multiple antigens other than hHER2 are recognized by the immune system in mice cured of E0771-hHER2 tumors upon RC48 and PD-1/PD-L1 treatments. Thus, the combination treatment induces T cells that recognize not only hHER2 but also other antigens of tumor cells.

\section{Discussion}

To investigate the direct effect of different agents on humanized targets within tumor cells, several studies have been conducted on immunocompromised mouse models (xenograft mouse models) ${ }^{21-25}$. In such experiments, the importance of the immune system, especially $T$ and B cells, was tended to be overlooked. In our study, we examined the immunologic effect of RC48 using an immunocompetent mouse model by introducing a hHER2 expressed murine cancer cell line. Furthermore, to study PD-1/PD-L1 signal blockade, humanized antibodies such as pembrolizumab and atezolizumab instead of murine antibodies were utilized in this study. Our PD-1 humanized transgenic mouse is a good model for achieving this goal. Transplanting human peripheral blood mononuclear cells into nude mice is another available model that used to evaluate drug combinations in human cancer cell lines in vivo (data not shown) ${ }^{26,27}$. Taking advantage of our transgenic model, we aimed to specifically dissect the immune-modulatory capacities of RC48, and subsequently its interaction with immunotherapy.

Previous studies have indicated that ADCs can induce antitumor immune activity upon intracellular release of the cytotoxic payload ${ }^{6-10}$. In other words, when ADC drug molecules arrive at the target site, they are enriched on the tumor surface, exerting a direct killing effect on the tumor or bystanders, greatly improving immune responses. For example, ado-trastuzumab emtansine (T-DM1) increased the number of tumor-infiltrating lymphocytes in human primary breast cancers and induced infiltration by effector $T$ cells in murine breast tumors $^{28}$. In addition, RC48 reduces the number of tumor cells, thereby minimizing the chronic exposure of T cells to tumor-derived antigens, which may render them nonfunctional. We therefore propose that the ADC with its cytotoxic payload reinstates immunosurveillance by driving a distinct DC maturation program, and subsequently T cell infiltration, whereas PD-1-blocking therapy potentially reinvigorates exhausted T cells $\mathrm{s}^{20,29,30}$.

Furthermore, monotherapies of PD-1/PD-L1 antibodies have demonstrated substantial clinical activity in different tumors ${ }^{31-38}$. However, only a limited number of patients respond to PD-(L)1 blockade, likely those whose tumors are pre-infiltrated by T cells. Others have reported that PD-L1 is upregulated in cancer cells and 
tumors after treatment with immunotherapy-based agents. Whereas DS-8201a demonstrates immune-modulating activity, the expression of PD-L1, an immune-inhibiting molecule, is increased during DS-8201a treatment; therefore, new treatments are needed. One solution to this problem is combination therapy ${ }^{20,30}$, such as with HER2-targeting therapy and blockade of the PD-1/PD-L1 inhibitory pathway, which has been tested in mouse models and are currently being evaluated in clinical trials (NCT02318901, NCT02605915, NCT01896999, NCT02581631, NCT02684292, NCT02572167). For combinations with immune checkpoint inhibitors, ADCs are considered superior to other chemotherapeutic agents because ADCs work selectively at the tumor site with reduced side effects in other organs.

In our model, combining RC48 treatment with PD-1/PD-L1 antibodies resulted in complete inhibition and greatly enhanced $T$ cell responses, leading to complete tumor rejection and memory formation ${ }^{28,39}$. Our data strongly support $T$ cell dependence and the efficacy of combination therapy, which is achieved through non-redundant but complementary mechanisms, i.e., RC48 augments $\mathrm{T}$ cell infiltration into the tumor by inducing tumor-specific, adaptive antitumor immunity, whereas PD-1 blockade reinvigorates exhausted T cells. Similar therapeutic benefits have been observed with other chemotherapies ${ }^{40,41}$. Notably, we used suboptimal yet therapeutically active doses of RC48, indicating that immune checkpoint inhibitors can boost the antitumor activity of ADCs, leading to a better tolerability of ADCs at sub-curative doses. More importantly, the synergistic effects of RC48 ADC with immune checkpoint inhibitors may lead to a reduction in the dosing regimen of both therapeutic modalities below their required effective doses during monotherapy. This can be expected to lead to a reduction in dose-limiting toxicities of individual treatments.

Mechanisms underlying the immunologic memory effect may rely on a series of processes including cell death, activation of DCs, T-cell activation, and possibly others. We found that the RC48 and PD-1/PD-L1 combination treatment contributed to immune memory formation. This study provides insights into the mechanisms underlying RC48 therapeutic activity and a rationale for potential therapeutic combination strategies with immunotherapy. Our data suggest that the PD-1/PD-L1 pathway inhibits activation of the immune system by RC48, where the anti-PD-1 antibody blocks the PD-1/PD-L1 inhibitory signal, increasing the efficacy of RC48. Therefore, the benefits of a combination therapy of RC48 with anti-PD-1/PD-L1 antibodies are strongly supported and this treatment modality is expected to be a new effective treatment for HER2-positive tumors.

\section{Acknowledgements}

The authors thank RemeGen, Ltd. for RC48 preparation.

\section{Disclosure}

Jianmin Fang reports being a shareholder of RemeGen Ltd., outside the submitted work. The authors report no other potential conflicts of interest in this work.

\section{References}

1 Su, C. Y. et al. Bispecific antibodies (anti-mPEG/anti-HER2) for active tumor targeting of docetaxel (DTX)-loaded mPEGylated nanocarriers to enhance the chemotherapeutic efficacy of HER2-overexpressing tumors. Drug Deliv 25, 1066-1079, doi:10.1080/10717544.2018.1466936 (2018). 
Yu, S. et al. Development and clinical application of anti-HER2 monoclonal and bispecific antibodies for cancer treatment. Exp Hematol Onco/6, 31, doi:10.1186/s40164-017-0091-4 (2017).

3 Parakh, S. et al. Evolution of anti-HER2 therapies for cancer treatment. Cancer Treat Rev 59, 1-21, doi:10.1016/j.ctrv.2017.06.005 (2017).

4 Loibl, S. \& Gianni, L. HER2-positive breast cancer. The Lancet 389, 2415-2429, doi:10.1016/s0140-6736(16)32417-5 (2017).

5 Prat, A., Pascual, T. \& Adamo, B. Intrinsic molecular subtypes of HER2+ breast cancer. Oncotarget 8 , 73362-73363, doi:10.18632/oncotarget.20629 (2017).

6 Tolcher, A. W. Antibody drug conjugates: lessons from 20 years of clinical experience. Ann Oncol 27, 2168-2172, doi:10.1093/annonc/mdw424 (2016).

7 Lambert, J. M. \& Morris, C. Q. Antibody-Drug Conjugates (ADCs) for Personalized Treatment of Solid Tumors: A Review. Adv Ther 34, 1015-1035, doi:10.1007/s12325-017-0519-6 (2017).

8 Bakhtiar, R. Antibody drug conjugates. Biotechnol Lett 38, 1655-1664, doi:10.1007/s10529-016-2160-x (2016).

9 Staudacher, A. H. \& Brown, M. P. Antibody drug conjugates and bystander killing: is antigen-dependent internalisation required? Br J Cancer 117, 1736-1742, doi:10.1038/bjc.2017.367 (2017).

10 Borcoman, E. \& Le Tourneau, C. Antibody drug conjugates: the future of chemotherapy? Curr Opin Oncol 28, 429-436, doi:10.1097/CCO.0000000000000310 (2016).

11 Parslow, A. C., Parakh, S., Lee, F. T., Gan, H. K. \& Scott, A. M. Antibody-Drug Conjugates for Cancer Therapy. Biomedicines 4, doi:10.3390/biomedicines4030014 (2016).

12 Lambert, J. M. \& Chari, R. V. Ado-trastuzumab Emtansine (T-DM1): an antibody-drug conjugate (ADC) for HER2-positive breast cancer. Journal of medicinal chemistry 57, 6949-6964, doi:10.1021/jm500766w (2014).

13 Takegawa, N. et al. DS-8201a, a new HER2-targeting antibody-drug conjugate incorporating a novel DNA topoisomerase I inhibitor, overcomes HER2-positive gastric cancer T-DM1 resistance. International journal of cancer 141, 1682-1689, doi:10.1002/ijc.30870 (2017).

$14 \mathrm{Li}, \mathrm{H}$. et al. An anti-HER2 antibody conjugated with monomethyl auristatin $\mathrm{E}$ is highly effective in HER2-positive human gastric cancer. Cancer Biol Ther 17, 346-354, doi:10.1080/15384047.2016.1139248 (2016).

15 Yao, X. et al. A novel humanized anti-HER2 antibody conjugated with MMAE exerts potent anti-tumor activity. Breast Cancer Res Treat 153, 123-133, doi:10.1007/s10549-015-3503-3 (2015).

16 von Minckwitz, G. et al. Trastuzumab Emtansine for Residual Invasive HER2-Positive Breast Cancer. The New England journal of medicine 380, 617-628, doi:10.1056/NEJMoa1814017 (2019).

17 Hunter, F. W. et al. Mechanisms of resistance to trastuzumab emtansine (T-DM1) in HER2-positive breast cancer. Br J Cancer 122, 603-612, doi:10.1038/s41416-019-0635-y (2020).

18 Muller, P. et al. Microtubule-depolymerizing agents used in antibody-drug conjugates induce antitumor immunity by stimulation of dendritic cells. Cancer immunology research 2, 741-755, doi:10.1158/2326-6066.CIR-13-0198 (2014).

19 Berger, G. K. et al. Brentuximab vedotin for treatment of non-Hodgkin lymphomas: A systematic review. Critical reviews in oncology/hematology 109, 42-50, doi:10.1016/j.critrevonc.2016.11.009 (2017).

20 Gerber, H. P., Sapra, P., Loganzo, F. \& May, C. Combining antibody-drug conjugates and immune-mediated cancer therapy: What to expect? Biochem Pharmacol 102, 1-6, doi:10.1016/j.bcp.2015.12.008 (2016).

21 Ashizawa, T. et al. Antitumor Effect of Programmed Death-1 (PD-1) Blockade in Humanized the NOG-MHC Double Knockout Mouse. Clin Cancer Res 23, 149-158, doi:10.1158/1078-0432.CCR-16-0122 (2017).

22 Lin, S. et al. Establishment of peripheral blood mononuclear cell-derived humanized lung cancer mouse models for studying efficacy of PD-L1/PD-1 targeted immunotherapy. mAbs 10, 1301-1311, 
doi:10.1080/19420862.2018.1518948 (2018).

23 Morton, J. J., Bird, G., Refaeli, Y. \& Jimeno, A. Humanized Mouse Xenograft Models: Narrowing the Tumor-Microenvironment Gap. Cancer Res 76, 6153-6158, doi:10.1158/0008-5472.CAN-16-1260 (2016).

24 Sanmamed, M. F. et al. Nivolumab and Urelumab Enhance Antitumor Activity of Human T Lymphocytes Engrafted in Rag2-/-IL2Rgammanull Immunodeficient Mice. Cancer Res 75, 3466-3478, doi:10.1158/0008-5472.CAN-14-3510 (2015).

25 Pyo, K. H. et al. Promising preclinical platform for evaluation of immuno-oncology drugs using Hu-PBL-NSG lung cancer models. Lung cancer 127, 112-121, doi:10.1016/j.lungcan.2018.11.035 (2019).

26 Burova, E. et al. Characterization of the Anti-PD-1 Antibody REGN2810 and Its Antitumor Activity in Human PD-1 Knock-In Mice. Molecular cancer therapeutics 16, 861-870, doi:10.1158/1535-7163.MCT-16-0665 (2017).

27 Wang, M. et al. Humanized mice in studying efficacy and mechanisms of PD-1-targeted cancer immunotherapy. FASEB journal : official publication of the Federation of American Societies for Experimental Biology 32, 1537-1549, doi:10.1096/fj.201700740R (2018).

28 Muller, P. et al. Trastuzumab emtansine (T-DM1) renders HER2+ breast cancer highly susceptible to CTLA-4/PD-1 blockade. Sci Trans/ Med 7, 315ra188, doi:10.1126/scitranslmed.aac4925 (2015).

29 Checkpoint Inhibitors Boost Power of Antibody-Drug Conjugate. Cancer Discov 6, OF3, doi:10.1158/2159-8290.CD-NB2015-172 (2016).

30 Sharma, P. \& Allison, J. P. Immune checkpoint targeting in cancer therapy: toward combination strategies with curative potential. Cel/ 161, 205-214, doi:10.1016/j.cell.2015.03.030 (2015).

31 Callahan, M. K. \& Wolchok, J. D. At the bedside: CTLA-4- and PD-1-blocking antibodies in cancer immunotherapy. Journal of leukocyte biology 94, 41-53, doi:10.1189/jlb.1212631 (2013).

32 Tsai, H. F. \& Hsu, P. N. Cancer immunotherapy by targeting immune checkpoints: mechanism of T cell dysfunction in cancer immunity and new therapeutic targets. Journal of biomedical science 24, 35, doi:10.1186/s12929-017-0341-0 (2017).

33 Ott, P. A., Hodi, F. S., Kaufman, H. L., Wigginton, J. M. \& Wolchok, J. D. Combination immunotherapy: a road map. J Immunother Cancer 5, 16, doi:10.1186/s40425-017-0218-5 (2017).

34 Chen, D. S. \& Mellman, I. Elements of cancer immunity and the cancer-immune set point. Nature 541, 321-330, doi:10.1038/nature21349 (2017).

35 Okazaki, T. \& Honjo, T. PD-1 and PD-1 ligands: from discovery to clinical application. International immunology 19, 813-824, doi:10.1093/intimm/dxm057 (2007).

36 Balar, A. V. \& Weber, J. S. PD-1 and PD-L1 antibodies in cancer: current status and future directions. Cancer immunology, immunotherapy: C// 66, 551-564, doi:10.1007/s00262-017-1954-6 (2017).

37 Alsaab, H. O. et al. PD-1 and PD-L1 Checkpoint Signaling Inhibition for Cancer Immunotherapy: Mechanism, Combinations, and Clinical Outcome. Frontiers in pharmacology 8, 561, doi:10.3389/fphar.2017.00561 (2017).

38 Wang, Y., Wu, L., Tian, C. \& Zhang, Y. PD-1-PD-L1 immune-checkpoint blockade in malignant lymphomas. Annals of Hematology 97, 229-237, doi:10.1007/s00277-017-3176-6 (2017).

39 Iwata, T. N. et al. A HER2-Targeting Antibody-Drug Conjugate, Trastuzumab Deruxtecan (DS-8201a), Enhances Antitumor Immunity in a Mouse Model. Molecular cancer therapeutics 17, 1494-1503, doi:10.1158/1535-7163.MCT-17-0749 (2018).

40 Haratani, K. et al. U3-1402 sensitizes HER3-expressing tumors to PD-1 blockade by immune activation. The Journal of clinical investigation 130, 374-388, doi:10.1172/JCl126598 (2020).

41 D'Amico, L. et al. A novel anti-HER2 anthracycline-based antibody-drug conjugate induces adaptive anti-tumor immunity and potentiates PD-1 blockade in breast cancer. J Immunother Cancer 7, 16, doi:10.1186/s40425-018-0464-1 (2019). 


\section{Figures and legends}

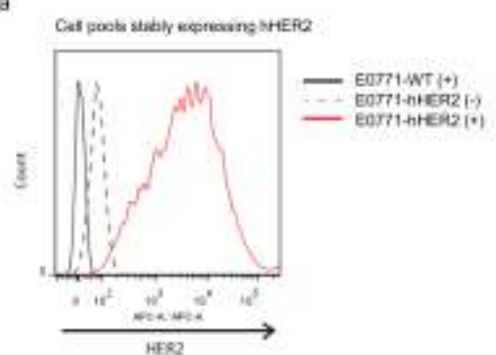

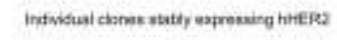

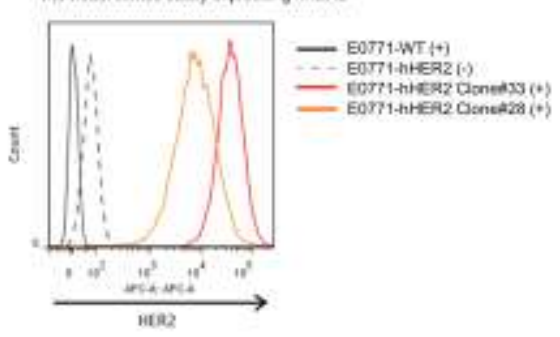

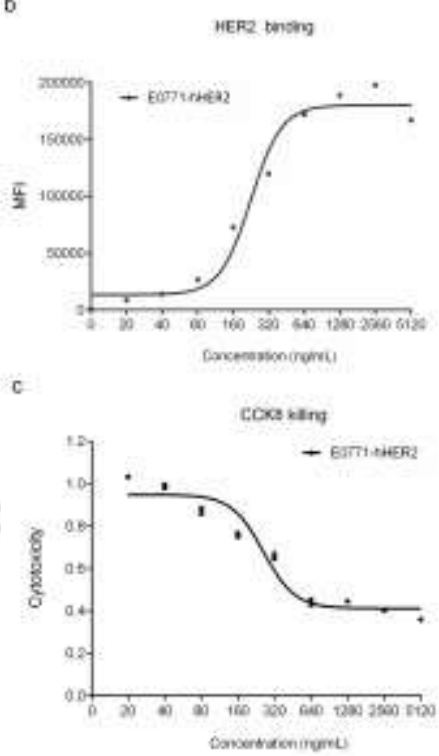

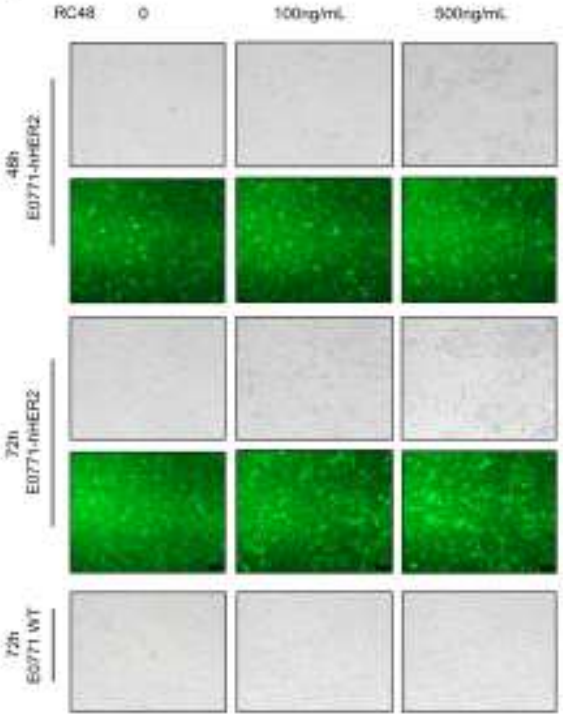

Fig. 1. Antitumor effect of RC48 in a hHER2+ mouse cancer cell.

a) Expression of exogenously introduced human her2 gene was confirmed in E0771 cells. Individual clones were isolated by single-cell sorting using flow cytometry.

b) Her2 binding ability of E0771-hHER2 (clone\#33) using disitamab $(1 \mu \mathrm{g} / \mathrm{mL})$.

c) CCK-8 assay for determining cell viability of E0771-hHER2 after RC48 treatment (clone\#33).

d) RC48 displays target-specific cell death properties. Images of E0771-hHER2 and E0771-WT cells after treatment with $\operatorname{RC} 48(0,100,500 \mathrm{ng} / \mathrm{mL})$ for 48 and $72 \mathrm{~h}$. Because the zsGreen1 fluorescence gene is also carried in the human her2 gene-containing vector, the tumor cells are shown in green. 
a

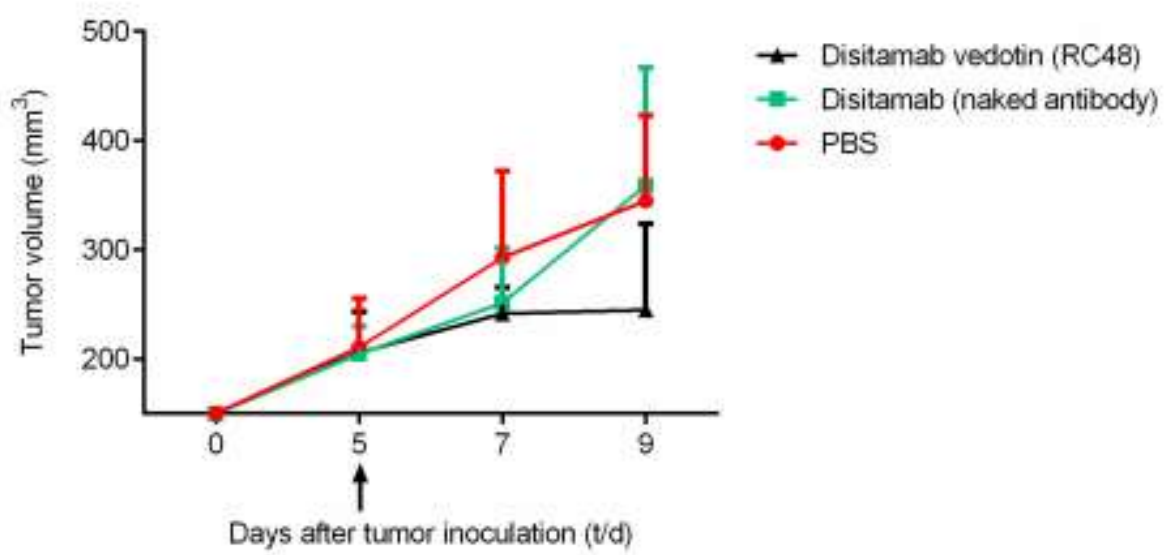

b
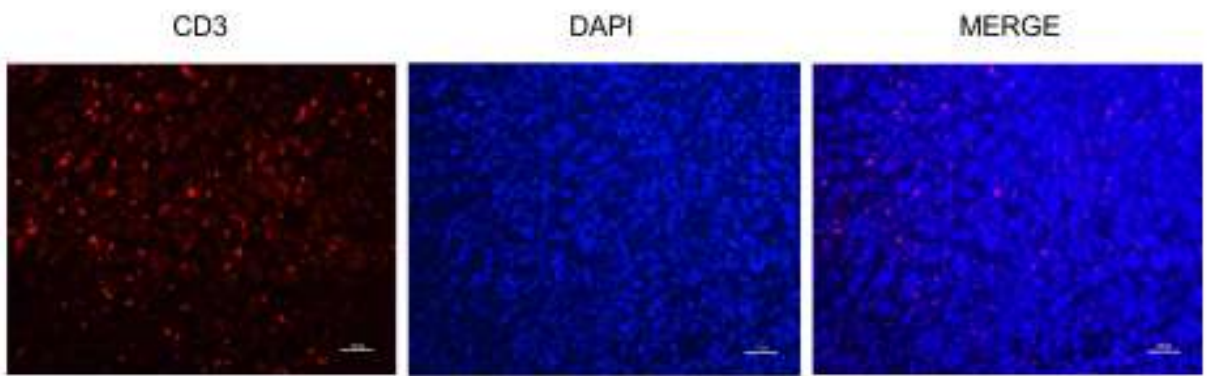

Disitamab
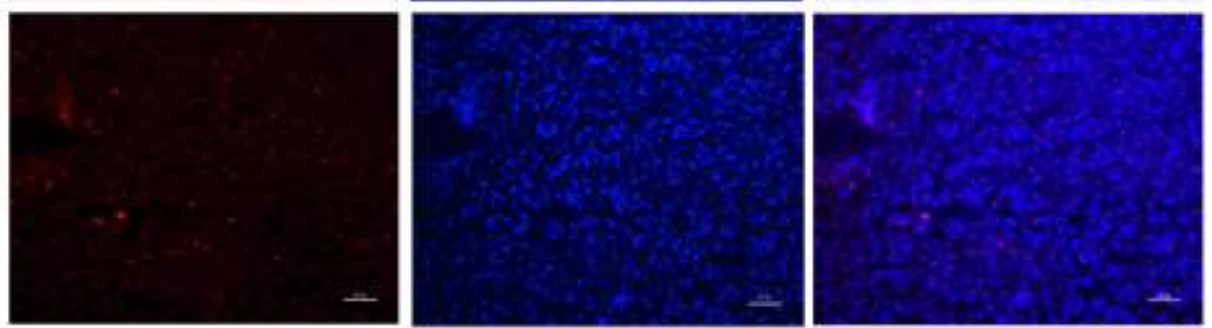

PBS
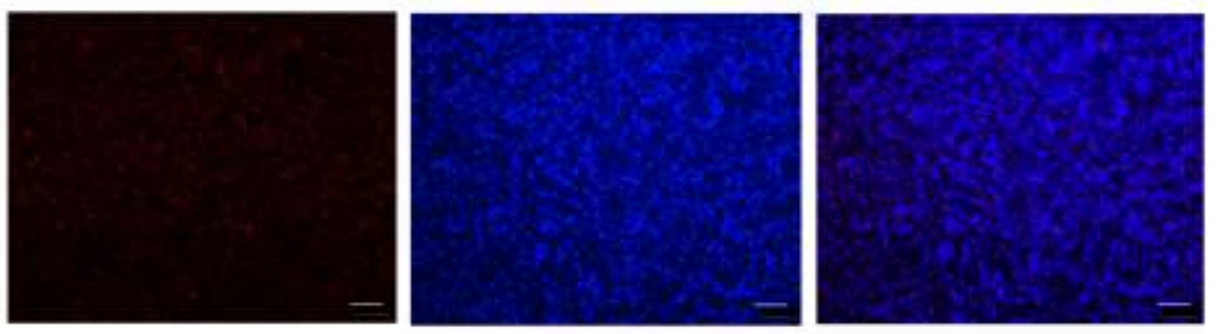

Fig. 2. RC48 strongly affects tumor growth in hHER2 xenograft breast cancer model and increases $T$ cell infiltration.

a) Antitumor effect was examined in a syngeneic mouse model with E0771-hHER2 cells (s.c. inoculation) upon treatment with anti-hHER2 antibody or RC48 (10 mg/kg, once, i.v.) at the time points indicated by arrow. The graph shows mean tumor volumes and standard errors $(n=5)$.

b) On day 9, mice were euthanized and isolated tumors were stained with CD3 antibody for T cells (red) and DAPI. Bar $=50 \mu \mathrm{m}$. 


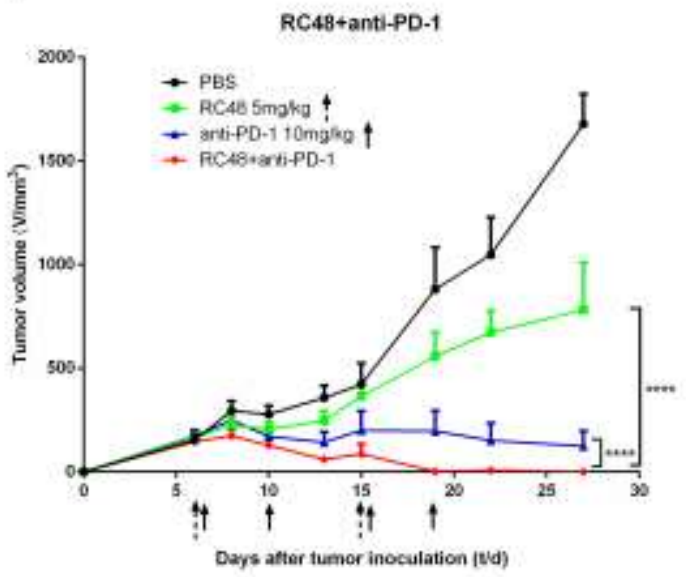

b

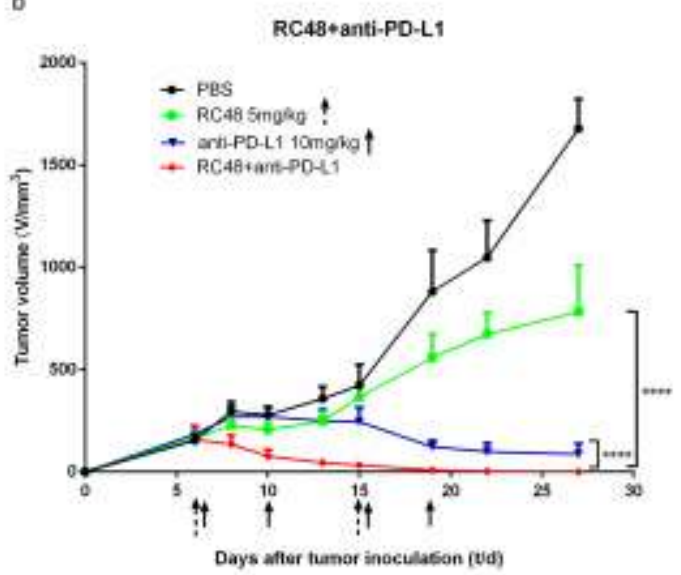

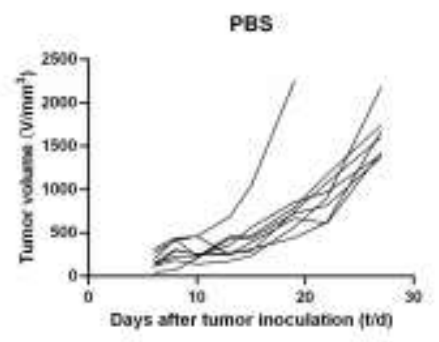

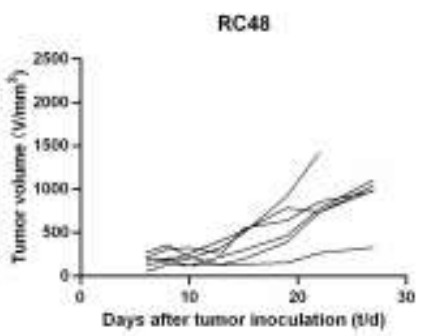

anti-PD-1

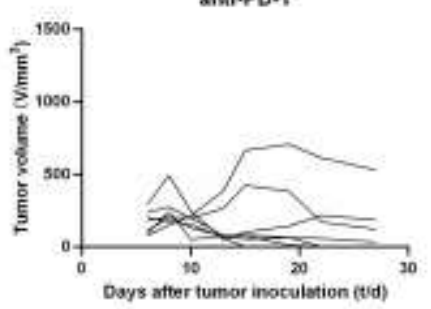

anti-PD.L1

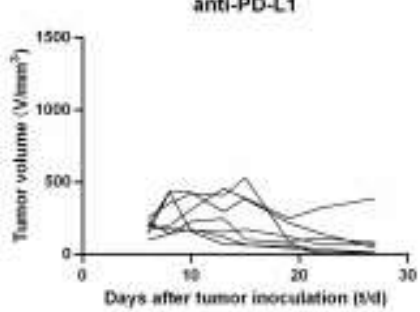

RC48+anti-PD-1

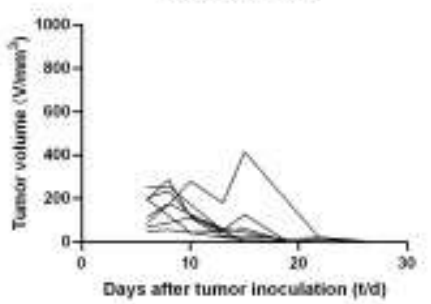

RC48+anti-PD-L1

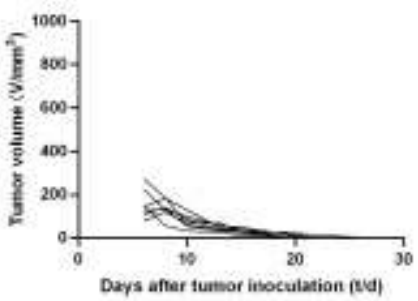

Fig. 3. Antitumor activity of immune checkpoint inhibitors and RC48 combination therapy.

a) Therapeutic response of E0771-hHER2 xenografted mice with a tumor volume of 150-200 $\mathrm{mm}^{3}$ following treatment with vehicle (PBS), RC48 alone (5 mg/kg, once a week, twice, i.v.), anti-PD-L1 alone (15 mg/kg, twice a week, 2 cycles, i.v.), or their combination (RC48 + anti-PD-L1). Compounds were administrated at the time points indicated by arrows.

b) Therapeutic response of of E0771-hHER2 animals with a tumor volume of $150-200 \mathrm{~mm}^{3}$ following treatment with vehicle (PBS), RC48 alone ( $5 \mathrm{mg} / \mathrm{kg}$, once a week, twice, i.v.), anti-PD-1 alone (10 mg/kg, twice a week, 2 cycles, i.v.), or their combination (RC48 + anti-PD-1). Statistical significance was analyzed by two-way ANOVA, $* * * \star p<0.0001$.

c) Individual tumor growth curve of the indicated treatments in $A$ and $B$. 

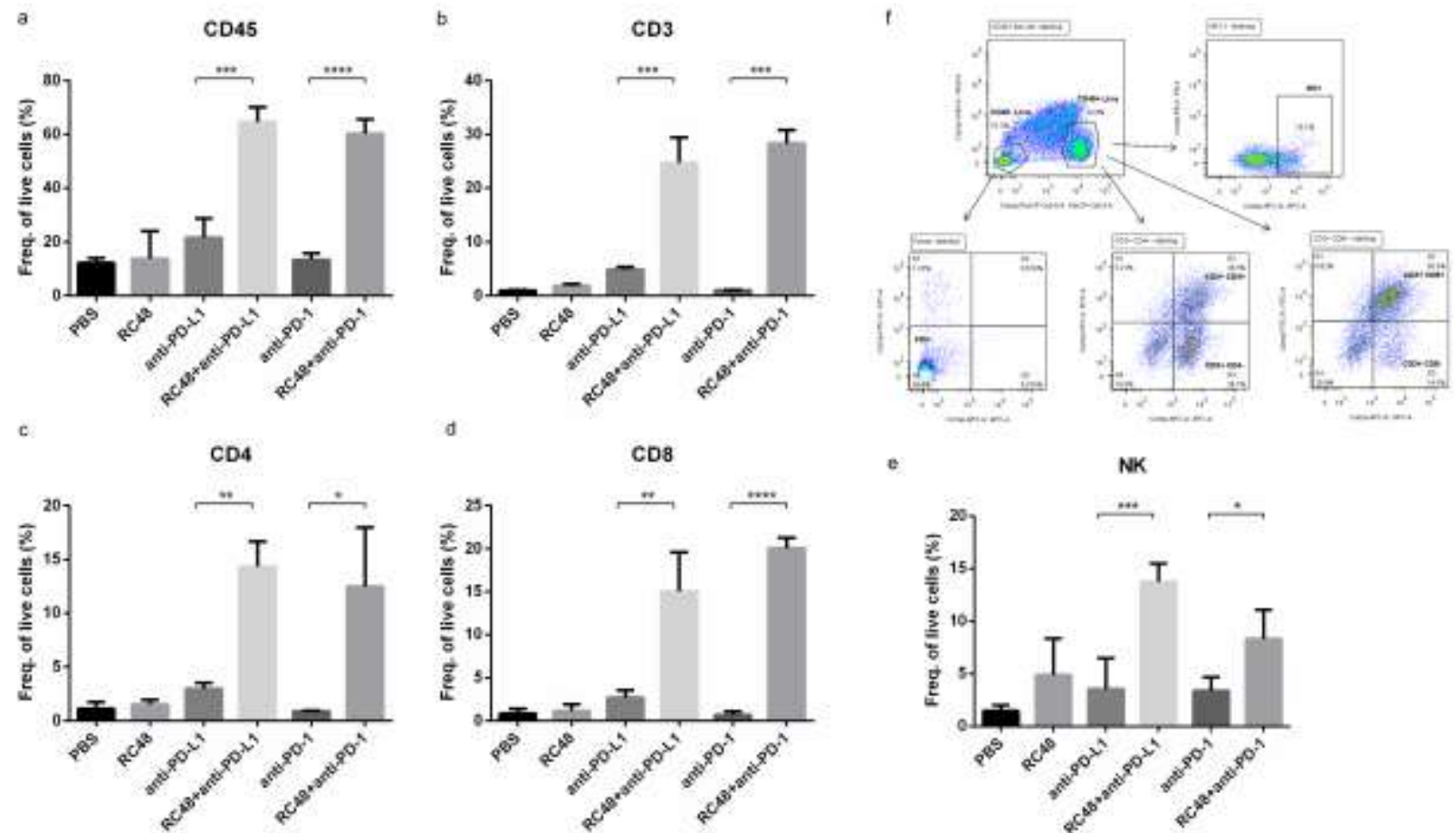

d

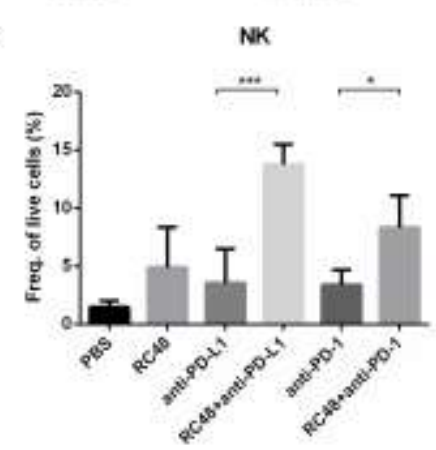

9
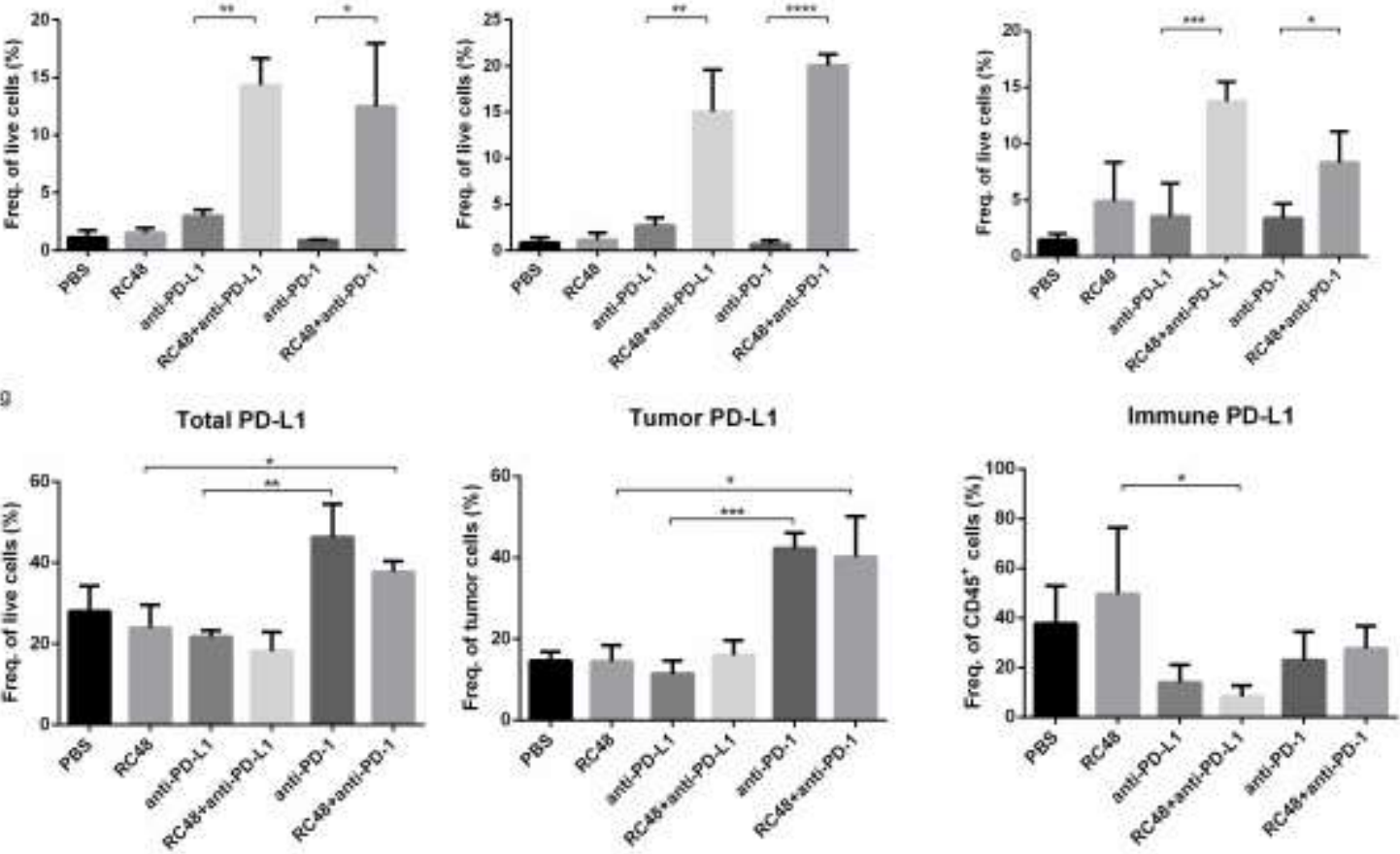

Fig. 4. Proliferation and activation of $\mathrm{CD} 4+$ and $C D 8+T$ cells infiltrating murine breast tumors.

a-e) Single-cell suspensions from the excised E0771 tumors treated as indicated were stained for immune cell markers CD45, CD3, CD4, CD8 for T cells and NK1.1 for NK cells according to the analysis method shown in f). g) Single-cell suspensions from the excised E0771 tumors were stained for the immune checkpoint marker PD-L1. Analysis of the PD-L1 expression on tumor cells, immune cells, and total live cells, respectively. Statistical significance was analyzed by an unpaired two-tailed $t$-test, $\star p<0.05, \star \star p<0.01, \star \star \star p<0.001$, and $\star \star \star \star \mathrm{p}<0.0001$. 


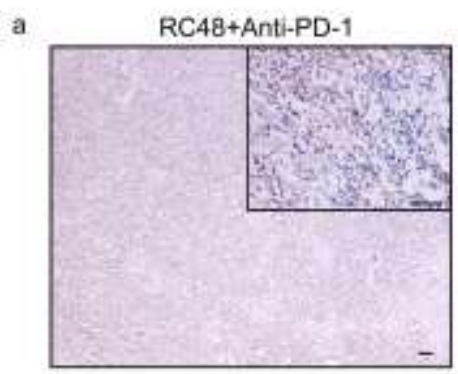

Anti-PD-1

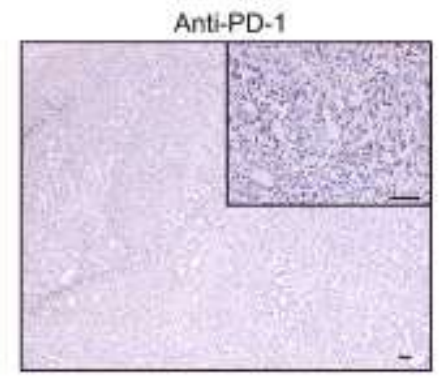

RC48

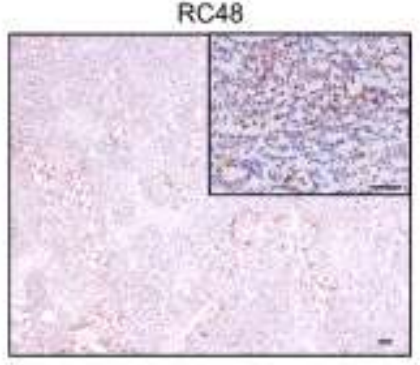

RC48+Anti-PD-L1

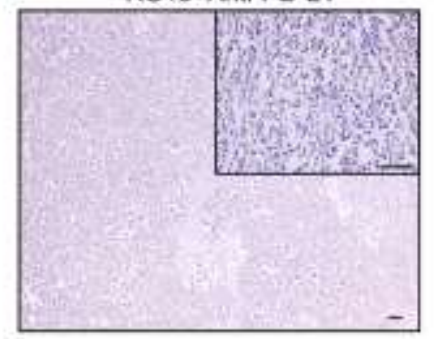

Anti-PD-L1

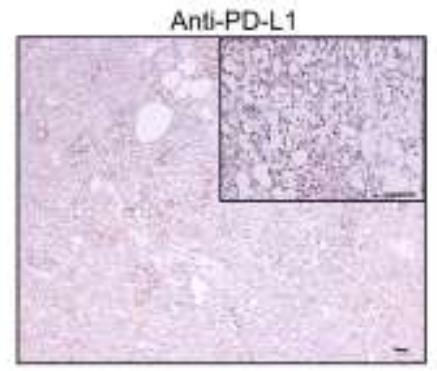

PBS

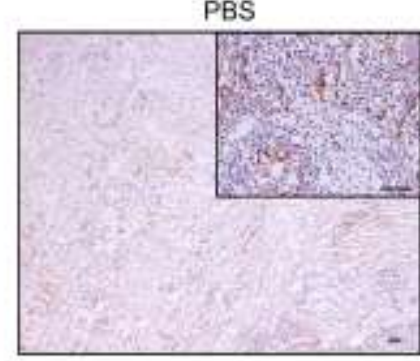

b

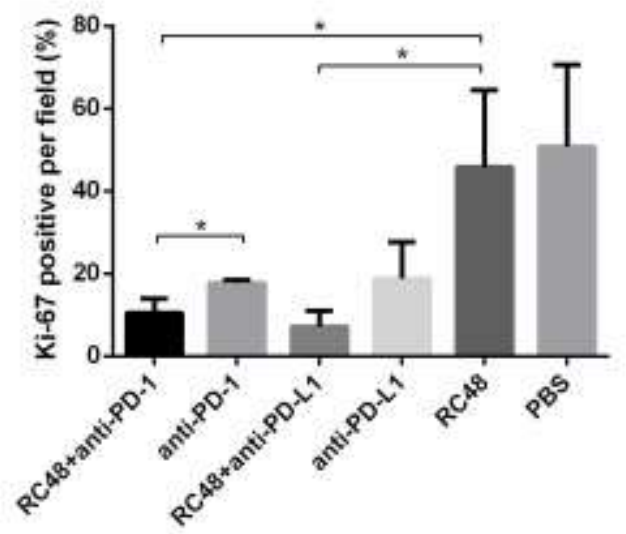

Fig. 5. Tumor immunostaining analysis after RC48 + PD-1/PD-L1 combined therapy.

a) Representative tumor sections were immunohistochemical stained of Ki67 for the indicated treatments. Scale bar $=100 \mu \mathrm{m}$.

b) Analysis of Ki67 positive field for the indicated treatments. Statistical significance was analyzed by an unpaired two-tailed $t$-test, $* \mathrm{p}<0.05$. 
a

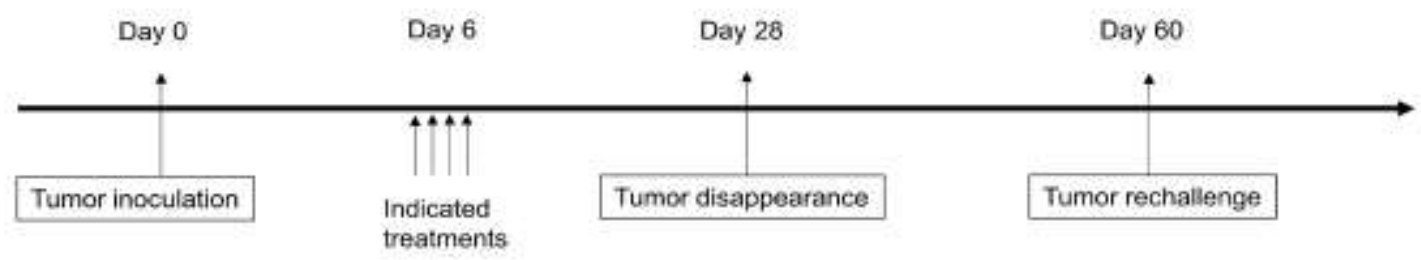

b

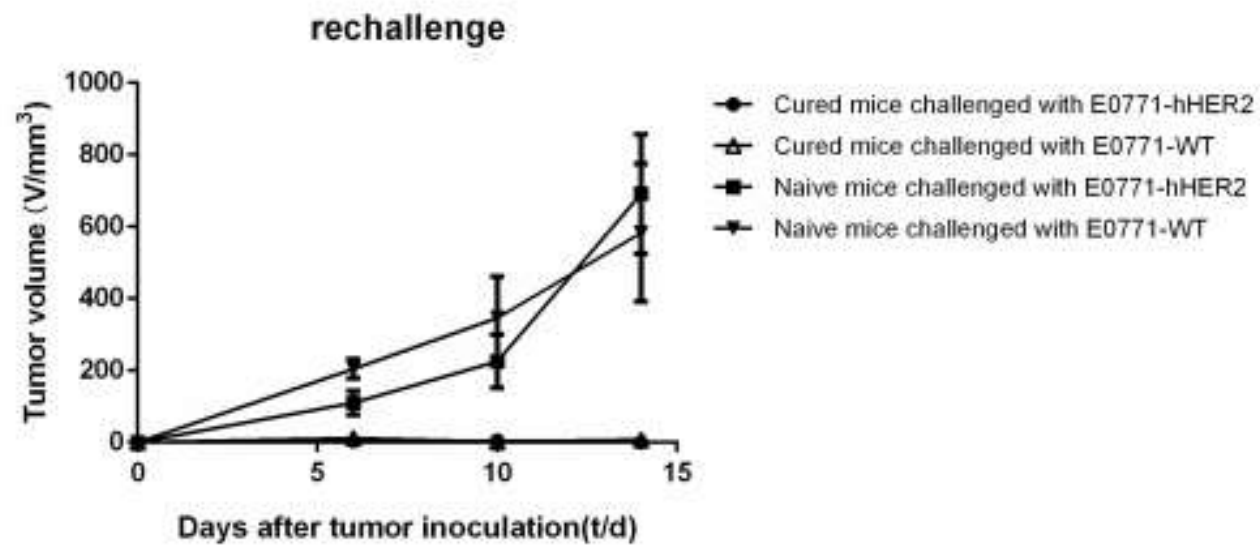

Fig. 6. RC48 and PD-1/PD-L1 combination therapy affords long-lasting immune protection.

a) Outline of a representative rechallenge experiment.

b) E0771-hHER2 tumor-free animals after ADC and PD-1/PD-L1 combination treatment were rechallenged with E0771-hHER2 and E0771-WT cancer cells. Tumor growth curve of the indicated groups. 
Figure S1

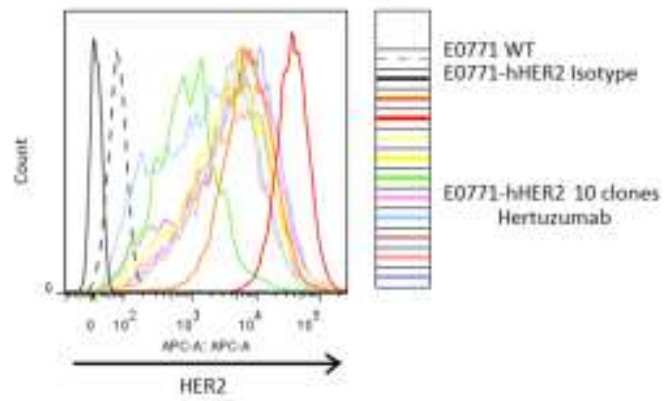

Figure S2
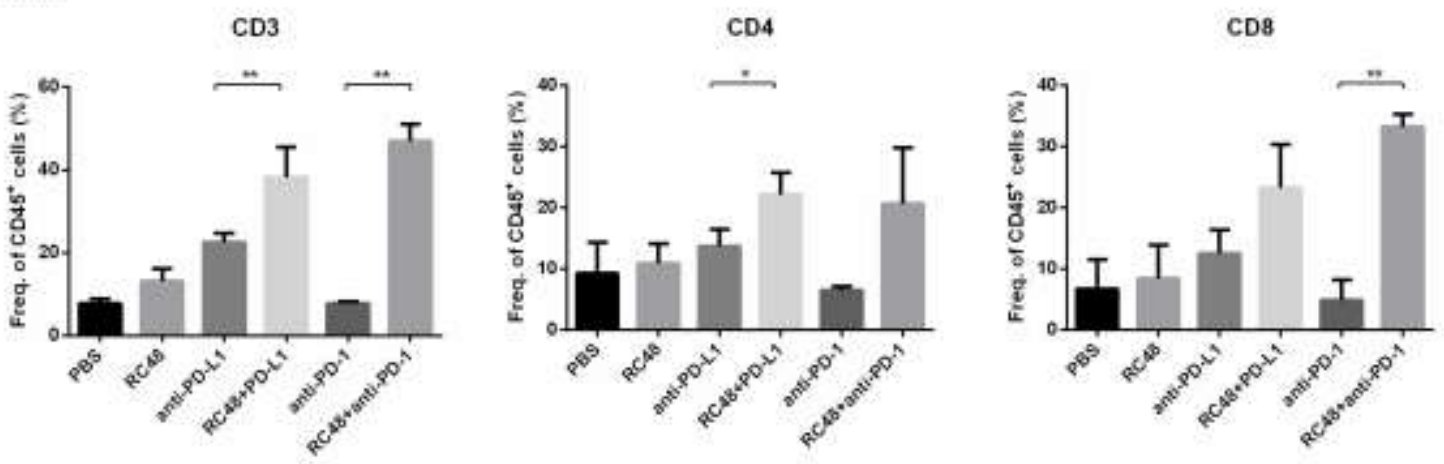

Figure S3

\begin{tabular}{|c|c|c|c|c|c|c|c|c|c|c|c|c|c|c|c|c|c|c|c|c|c|c|}
\hline Curna & $\begin{array}{l}\text { RCest } \\
\text { pot }\end{array}$ & 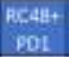 & $\begin{array}{c}\mathrm{HC} 4 \mathrm{~g}+ \\
\mathrm{pot}\end{array}$ & $\begin{array}{l}\text { RCAst } \\
\text { pot: }\end{array}$ & \begin{tabular}{|l|} 
SCAS: \\
POLt
\end{tabular} & $\begin{array}{l}\text { Rects- } \\
\text { pout }\end{array}$ & $\begin{array}{l}\text { Rest: } \\
\text { sout }\end{array}$ & $\begin{array}{l}\text { Medit } \\
\text { poi }\end{array}$ & \begin{tabular}{|c|}
$R 045+1$ \\
$P 011$
\end{tabular} & \begin{tabular}{|l|} 
RCABt- \\
Pos
\end{tabular} & \begin{tabular}{|l|} 
gedst \\
pout
\end{tabular} & \begin{tabular}{|l|} 
HCCAt: \\
pout
\end{tabular} & $\begin{array}{l}\text { nesest } \\
\text { PoLI }\end{array}$ & \begin{tabular}{|l|} 
BCALt: \\
pou1
\end{tabular} & Naive & $N=N_{0}$ & ratere & Raive & Nariven & Naiven & Raine & Naine \\
\hline no & 22 & 75 & 29 & 34 & 38 & 40 & 61 & 24 & 26 & 56 & 42 & 54 & 57 & 62 & 1 & 2 & 3 & 4 & 5 & 6 & 7 & 8 \\
\hline $\begin{array}{l}\text { Rechallengs } \\
\text { Dory } 0\end{array}$ & \multicolumn{7}{|c|}{ EOTT2-hHER2 } & \multicolumn{7}{|c|}{ EOTIL-WT } & \multicolumn{4}{|c|}{ EOT71-hHER2 } & \multicolumn{4}{|c|}{ EOT/1-WT } \\
\hline Dosy 6 & 10 由 & $14 \%$ & ㅁ. & ㅁ & of & 0 & & 135 & 14 & 135 & 1755 & 135. & & c) 1228 & | 67.194 & 157.91 & 17627 & \$251 & 28006 & 27567 & 17675 & 15791 \\
\hline Dar 10 & 20.42 & 19.23 & 0 & 0 & a. & 0 & 0 & 0 & 0 & 0 & 0 & 135 & E & 0 o 0 & 11459 & 27652 & 41106 & 100.8 & 26701 & 688.1 & 158.77 & 27652 \\
\hline Day 14 & 0 & & o & 0 & 0 & 0 & 0 & & 19.45 & 0 & 0 & 35.7 & 0 & of & 667.39 & 855198 & 100419 & 236.7 & 286624 & 2001.3 & 324891 & 17552 \\
\hline
\end{tabular}

